\title{
Complementing EMR-based Interventions to Improve Hepatitis C Screening
}

\author{
Rebecca J. Fisk ${ }^{1}$, Disha Kumar ${ }^{2}$, Daniel R. Murphy ${ }^{3}$, Monisha Arya ${ }^{3}$ \\ 'University of Texas- Houston Health Sciences Center School of Public Health, Houston, Texas, USA; \\ ${ }^{2}$ Baylor College of Medicine, Houston, Texas, USA; \\ ${ }^{3}$ Department of Medicine, Baylor College of Medicine, Houston, Texas, USA
}

To the Editor,

We read with interest the recent publication by Yeboah-Korang et al. titled "Hepatitis C screening in commercially insured US birth-cohort patients: Factors associated with testing and effect of an EMR-based screening alert." ${ }^{[1]}$ We were pleased to read efforts of Yeboah-Korang et al. to improve HCV testing for the 1945-1965 birth cohort in accordance with national HCV screening recommendations. Although the authors found that a physician-facing electronic medical record (EMR) bestpractice alert (BPA) increased $\mathrm{HCV}$ testing a significant amount, the rate of testing after implementation remained low at $10 \%$. This suggests that patient-directed approaches could complement physician-directed EMR BPAs to achieve national HCV screening recommendations.

Physicians believe that having patients who are educated about HCV would help physicians better adhere to the HCV screening recommendations. ${ }^{[2]}$ National campaigns, such as those from the Centers for Disease Control and Prevention and pharmaceutical companies, are attempting to increase awareness among birth cohort patients on the need to be HCV tested. We believe a timely, personalized HCV testing campaign directed at patients may complement existing physician-directed efforts, such as EMR prompts, to educate patients and help improve HCV testing in healthcare settings.

Text messaging may provide an efficient mode of communication to educate birth cohort patients of the need for $\mathrm{HCV}$ testing and is already used by $68 \%$ of clinics to deliver patient appointment reminders. ${ }^{[3]}$ Text messages could empower patients to ask their physicians about HCV testing. Unlike national media campaigns, text messages can be personalized with patients' names and be sent to the patient immediately before their clinic appointment to help them set the agenda for their appointment. Our own proof of concept study (data unpublished) of a text message campaign to improve HCV testing in primary care has shown acceptability, feasibility, and a promising trend for increasing testing rates.

A multifaceted approach is needed to augment HCV testing. Physician-based EMR prompts, such as the one implemented by Yeboah-Korang et al., have shown mixed results. ${ }^{[1,4,5]}$ We are optimistic that complementing physician-based BPAs with patient education and engagement would further improve HCV testing in healthcare settings.

\section{Conflict of Interest}

There is no conflict of interest declared.

\section{REFERENCES}

1. Yeboah-Korang A, Beig MI, Khan MQ, Goldstein JL, Macapinlac DM, Maurer D, et al. Hepatitis C screening in commercially insured U.S. birth-cohort patients: Factors associated with testing and effect of an EMR-based screening alert. J Transl Int Med 2018; 6:82-9.

2. Thomson M, Konerman MA, Choxi H, Lok AS. Primary Care Physician Perspectives on Hepatitis C Management in the Era of Direct-Acting Antiviral Therapy. Dig Dis Sci 2016;61:3460-8.

3. MGMA Stat. Text messaging remains an effective tool for patient appointment reminders [EB/OL]. Available from: https://www.mgma.com/data/ 
Fisk et al: : Interventions to improve hepatitis C screening

data-stories/text-messaging-remains-an-effective-tool-for-patientappointment-reminders_Accessed on August 15, 2018.

4. Nitsche B, Miller SC, Giorgio M, Berry CA, Muir A. Improving Hepatitis C Identification: Technology Alone Is Not the Answer. Health Promot Pract 2017;19:506-12.

5. Gemelas J, Locker R, Rudd S, Prevost C, Reilley B, Leston J. Impact of Screening Implementing HCV Screening of Persons Born 1945-1965: A Primary Care Case Study. J Prim Care Community Health 2015;7:30-2.
How to cite this article: Fisk RJ, Kumar D, Murphy DR, Arya M. Complementing EMR-based Interventions to Improve Hepatitis C Screening. J Transl Intern Med 2018; 6: 198-9. 